\title{
Macrocells-User Protected Interference-Aware Transmit Power Control for LTE-A Heterogeneous Networks
}

\author{
Arbab Waheed Ahmad, Heekwon Yang, Gul Shahzad, and Chankil Lee \\ Department of Electronics and Communication Engineering, Hanyang University, Ansan, Gyeonggi-do 429-791, Republic of Korea \\ Correspondence should be addressed to Chankil Lee; cklee@hanyang.ac.kr
}

Received 12 January 2016; Accepted 18 April 2016

Academic Editor: Alessandro Cilardo

Copyright ( 2016 Arbab Waheed Ahmad et al. This is an open access article distributed under the Creative Commons Attribution License, which permits unrestricted use, distribution, and reproduction in any medium, provided the original work is properly cited.

In Long Term Evolution-Advanced (LTE-A) heterogeneous networks (HetNets), small cells are deployed within the coverage area of macrocells having 1:1 frequency reuse. The coexistence of small cells and a macrocell in the same frequency band poses cross-tier interference which causes outage for macrocells users and/or small cell users. To address this problem, in this paper, we propose two algorithms that consider the received interference level at the evolved NodeB (eNB) while allocating transmit power to the users. In the proposed algorithm, the transmit power of all users is updated according to the target and instantaneous signal-to-noise-plusinterference ratio (SINR) condition as long as the effective received interference at the serving eNB is below the given threshold. Otherwise, if the effective received interference at the eNB is greater than the threshold, the transmit power of small cell users is gradually reduced in order to guarantee the target SINR for all macrocells users, aiming for zero-outage for macrocells users at the cost of an increased outage ratio for small cell users. Further, in the second algorithm, the transmit power of all users is additionally controlled by the power headroom report that considers the current channel condition while updating the transmit power which results in the outage ratio decreasing for small cell users. The extensive system-level simulations show significant improvements in the average throughput and outage ratio when compared with the conventional transmit power control technique.

\section{Introduction}

Long Term Evolution (LTE), developed by the Third Generation Partnership Project (3GPP), is an orthogonal frequencydivision multiplexing access- (OFDMA-) based mobile broadband wireless communication technology. In release 10, 3GPP introduced the advanced features of LTE, referred to as LTE-Advanced (LTE-A) [1]. Considering the need for increased data traffic, LTE-A is targeting low latency and high peak data rates at reduced costs. To achieve these targets, 3GPP has been focusing on various aspects of LTEA which include utilization of higher frequency bands, enhanced MIMO, cooperative multipoint communication, carrier aggregation, and deployment of heterogeneous networks (HetNets) [2]. Beside others, the concept of HetNets has attracted considerable attention due to its high potential to enhance system capacity and coverage [3]. Thus, HetNets, utilizing a diverse set of base stations, are considered good contenders for providing improvement in the spectral efficiency per unit area.

HetNets can be realized by deploying unplanned, lowpower small cells (femtocells, picocells, and relays) in traffic hotspots or coverage-challenged areas overlaid by a highpower planned macrocell [4]. As LTE-A is designed for a frequency reuse of one, these small cells use the same spectrum that is used by the overlaying macrocell. The deployment of small cell users within the coverage area of macrocell users (MUEs) brings harmful intertier interference as a by-product along with the capacity improvement. In LTEA homogeneous networks, conventional fractional power control scheme is used to mitigate the cochannel intercell interference [5]; however, in HetNet environment there is no proper frequency planning among tiers to cope with interference from small cell users and neighboring MUEs. This severe intertier/intercell interference affects the user's signal to interference plus noise ratio (SINR) which results 
in an increase in the users' outage ratio; for the most part, the users close to cell edges are mostly vulnerable to this harmful interference [6]. Since, the macrocell is deployed with high priority radio network and not for small cells; hence, MUEs entail higher priority of network coverage. So, it is essential to control interference from small cells and ensure protection of MUEs in heterogeneous deployment. Thus, there exists a strong need for intertier/intercell interference management for HetNets. Here, it is noticeable that intracell interference is mitigated by the orthogonality feature of OFDMA and the single-carrier frequency-division multiplexing access (SC-FDMA) used in the downlink and uplink of LTE-A, respectively [7]. Moreover, the major interference scenario of interest in this paper is the intertier interference from small cell users and intercell interference from macrocell users to the uplink traffic of the evolved NodeB (eNB) in a HetNet environment. In this work, we have used femto small cells because, unlike other small cell options, femtocells are user-deployed and can create a worse intertier interference scenario.

In this paper, we address the problem of intertier interference in HetNets through an interference-aware transmit power control (IA-TPC) method. Having obtained the interference limit of each user, we intend to develop an adaptive power control algorithm that should consider the total effective received interference at the eNB while allocating transmit power to all users with the aim of having all macro users reach their target SINR with a minimum outage ratio for femtocells users (FUEs). The existing interference management schemes do not assure the protection of MUEs from the harmful interference caused to MUEs by FUEs, which results in the outage of some MUEs. Still, these schemes can be used by FUEs provided that the interference caused by them to MUEs does not exceed a given threshold. In particular, if FUEs limit their transmit power level so that the total interference caused to MUEs does not exceed a given threshold, each MUE is able to reach its target SINR and FUEs can minimize their outage ratio. The major contributions of this work can be summarized as follows.

(i) We define the problem of transmit power control in the HetNet environment in order to maximize the throughput by attaining a zero-outage constraint for MUEs with as minimum an amount of outage as possible for FUEs. To achieve this goal, we present two algorithms, IA-TPC and power headroom report (PHR) based IA-TPC. In the proposed algorithms, the transmit power of all users is adjusted by properly following the effective target SINR. If the effective received interference at an $\mathrm{eNB}$ is above a given threshold, the FUEs adaptively decrease their transmit power to protect MUEs. Further, in the PHRbased IA-TPC algorithm, the transmit power is additionally adjusted by the PHR factor that considers the current channel condition, which results in reducing the outage ratio for FUEs.

(ii) Secondly, we prove that there always exists a valid power vector for the proposed algorithms; that is, in every interference scenario, MUEs achieve their target SINR.

(iii) Third, we have shown that with the proposed algorithms the average throughput of MUEs can be significantly improved while a slight improvement in the throughput of FUEs can be obtained by properly adjusting the transmit power using proposed algorithms.

(iv) Finally, we have compared the performance of the proposed algorithms with the conventional fractional power control (FPC) algorithm [5] and the outage performance shows the significance of the proposed algorithms.

The rest of this paper is organized as follows. The next section provides a literature overview of the related work. Section 3 presents the system model and states the problem. In Section 4, based on the received interference at the eNB, we introduce the proposed IA-TPC and PHR-based IATPC algorithms. Section 5 discusses the signaling overhead, valid power existence, and outage ratio for the proposed algorithms. In Section 6, the performance evaluation is discussed in detail and Section 7 concludes the paper.

\section{Literature Overview}

During the recent years, many of researchers including 3GPP paid full attention to the interference that HetNets have brought as a by-product together with capacity enhancement [6-22]. To combat the harmful interference in HetNets, different approaches have been studied in the literature. In [9, 10] the authors have proposed the fractional frequency reuse scheme that allocates different frequency bands to the MUEs and femtocells user equipment (FUEs) to avoid intertier interference in HetNets. Although the results showed an acceptable outage ratio, the proposed concept compromises on the available bandwidth and is somehow contradictory to the concept of $1: 1$ frequency reuse in HetNets. Similarly, in [11-13] the authors have proposed time muting, transmission time scheduling, and multiuser scheduling schemes to mitigate intertier interference; yet it requires the exchange of control information that needs extra signaling overhead resulting in inefficient spectrum usage along with complex computations. Further, in [12] the authors considered time scheduling and location information while allocating transmit power to FUEs. Scheduling nonneighboring UEs in same time slots results in reduced interference from neighboring UEs and an acceptable outage ratio; however, this performance is achieved at the cost of reduced throughput, increased complexity, and extra signaling overhead.

Besides, there are a few articles in the literature that have discussed power control to handle interference concerns [1422]. The intercell interference coordination (ICIC), enhanced ICIC, and further enhanced ICIC proposed by the 3GPP focused on power control techniques to combat intertier interference $[14,15]$. The authors in $[16,17]$ discussed openloop and closed-loop power control mechanisms with a fractional path loss compensation factor given by 3 GPP LTE. 
In [8], the authors identify the unique uplink interference patterns for LTE networks and propose two learning-based algorithms, driven by a set of measurement data, which update the cell-specific optimal power parameters. Though the authors achieved a substantial gain in overall throughput, it was at the cost of decreased MUE throughput. In [18], the authors used the conventional closed-loop FPC method to handle interference. The closed-loop FPC is proposed for LTE networks; thus, it does not consider the path loss and interference caused by small cells in a HetNet environment. Moreover, in [13], the transmit power is adjusted based on a high interference indicator (HII) parameter, yet the authors consider interference only from neighboring macrocells in the LTE environment. Similarly, in [19], the authors consider interference from macrocells while combating interference by dynamically controlling the transmit power. Recently, in [20], a distributed power allocation scheme was presented for HetNets where users adjust their power locally based on backhaul load variation. The algorithm presented in this work reduces the system complexity and is energy efficient; however, the authors do not consider the interference situation at the eNB; hence, in a densely deployed HetNet environment the overall performance of the system may decrease. In [21], the uplink power of FUEs is controlled in accordance with aggregate resource usage in order to maintain the throughput of MUEs. Similarly, in [22], an uplink power control algorithm based on fixed and dynamic thresholds of the interference is proposed in order to reduce intertier interference from FUEs to MUEs. Though this algorithm compensates for the throughput degradation of MUEs, it does not guarantee the protection of MUEs while adjusting the uplink power. Moreover, the outage of FUEs may not be controlled and FUE throughput may drastically decrease.

Unlike the existing literature, in this paper, we propose IA-TPC and PHR-based IA-TPC with reduced signaling overhead and computational complexity for HetNet environments. The objective of this work is to support the maximal number of FUEs subject to MUE protection. To the best of our knowledge, this is the first work that guarantees the protection of MUEs while combating the harmful interference in HetNet environment using power control technique.

\section{System Model and Problem Formulation}

3.1. System Model. We consider an LTE-A-based, two-tier HetNet system where femtocells are deployed within the coverage area of macrocells as shown in Figure 1. The overlaying macrocells share the whole available spectrum with the femtocells with a frequency reuse factor of one. The set of all macrocells is denoted by $N_{m}$ served by $B_{m}$ eNBs and there are $U_{m}$ MUEs in each macrocell. The set of all femtocells is denoted by $N_{f}$ served by $B_{f}$ femto eNBs (feNB) and there are $U_{f}$ FUEs in each femtocell. In order to generate the hotspot scenario for femtocell deployment, users are deployed according to the Poisson cluster process and each cluster contains one feNB. Further, it is assumed that FUEs are connected in the closed subscriber group (CSG) mode; that is, only specific registered users have access to connect with the feNB. We considered fixed femtocell deployment;

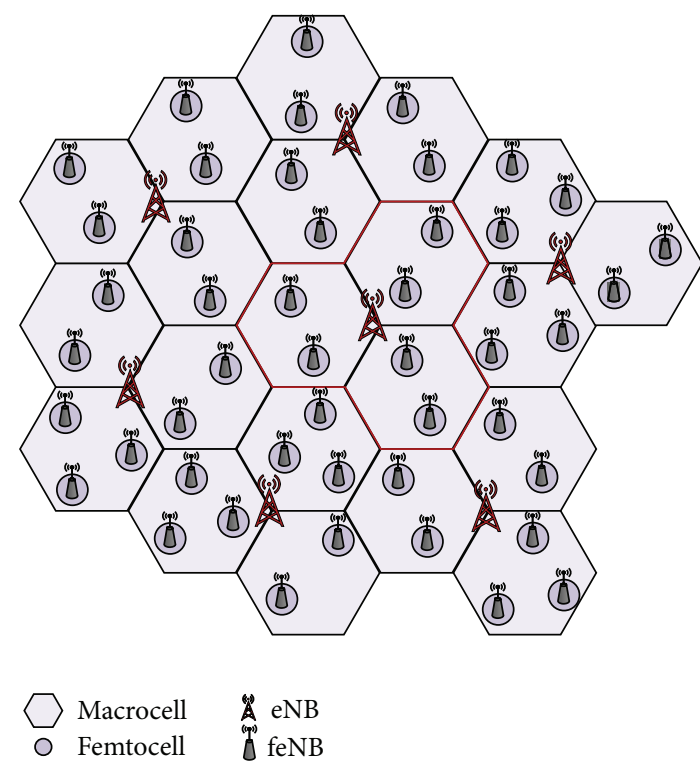

FIGURE 1: Heterogeneous network scenario.

that is, the feNB is stationary or moving at a very low speed (i.e., up to $3 \mathrm{~km} / \mathrm{hr}$ ). Interference management of moving HetNets is a future consideration and is beyond the scope of this work. We denote the set of MUEs associated with eNB $k \in B_{m}$ by $U_{m}^{k}$ and the set of FUEs associated with feNB $k \in B_{f}$ by $U_{f}^{k}$. The set of all users in a HetNet system can be denoted by $U=\sum_{n=1}^{N_{m}} U_{m n} \cup \sum_{p=1}^{N_{f}} U_{f p}$, while the set of all base stations can be denoted by $B=B_{m} \cup B_{f}$.

Let $p_{i}$ be the uplink transmit power of user $i$ and $0 \leq$ $p_{i} \leq \bar{p}_{i}$, where $\bar{p}_{i}$ is the maximum limit of transmit power for user $i, \forall i \in U$. The serving (f)eNB of user $i$ is denoted by $B_{i}$, and $\forall B_{i} \in B$ the path gain of user $i$ to the serving eNB $B_{i}$ is denoted by $G_{B_{i}, i}$. The path gain of any arbitrary user $j$ (which belongs to any neighboring macrocell of the serving cell of user $i, \forall j \in U_{m}, j \notin B_{i}$ ) at the serving (f)eNB $B_{i}$ of user $i$ is given by $G_{B_{i}, j}$. Similarly, the path gain of any arbitrary user $k$ (which belongs to any overlaid/neighboring femtocell of the serving cell of user $i, \forall k \in U_{f}, k \notin B_{i}$ ) at the serving (f)eNB $B_{i}$ of user $i$ is given by $G_{B_{i}, k}$. Thus, the total received power of users $j$ and $k$ at the serving (f)eNB $B_{i}$ of user $i$ can be calculated as $p_{j} G_{B_{i},}$ and $p_{k} G_{B_{i}, k}$. Consequently, the SINR of user $i$ can be expressed as

$$
\gamma_{i}(p)=\frac{p_{i} G_{B_{i}, i}}{I_{i}(p)+\sigma_{B_{i}}^{2}}, \quad \forall i \in U, B_{i} \in B,
$$

where $\sigma_{B_{i}}^{2}$ is additive white Gaussian noise power received at the receiver of user $i$. In addition, $I_{i}$ is the total interference caused to user $i$ at its receiver and is given by

$$
\begin{aligned}
I_{i}(p)=\sum_{j \in U_{m}, j \notin B_{i}} p_{j} G_{B_{i}, j}+\sum_{k \in U_{f}, k \notin B_{i}} p_{k} G_{B_{i}, k}, \\
\quad \forall B_{i} \in B, \quad j \in U_{m}, k \in U_{f}, \quad(j, k) \notin B_{i} .
\end{aligned}
$$


The effective interference for user $i$ is the ratio of total interference caused to user $i$ and the path gain to its serving (f)eNB, denoted by $\zeta_{i}$, and it can be expressed numerically as

$$
\begin{aligned}
\zeta_{i}=\frac{I_{i}(p)}{G_{B_{i}, i}}=\frac{\sum_{j \in U_{m}, j \notin B_{i}} p_{j} G_{B_{i}, j}+\sum_{k \in U_{f}, k \notin B_{i}} p_{k} G_{B_{i}, k}}{G_{B_{i}, i}}, \\
\forall i \in U, B_{i} \in B, \quad j \in U_{m}, k \in U_{f},(j, k) \notin B_{i} .
\end{aligned}
$$

The SINR vector is denoted by $\gamma=\left[\gamma^{m}, \gamma^{f}\right]$, where $\gamma^{m}$ and $\gamma^{f}$ are the SINRs of MUEs and FUEs, respectively. The effective SINR of user $i$ is denoted by $\Gamma_{i}$ and is defined as the ratio of signal power received at the (f)eNB to the total received power at the (f)eNB (i.e., the summation of signal power, interference power, and noise received at the (f)eNB):

$$
\begin{aligned}
& \Gamma_{i}(p) \\
& =\frac{p_{i} G_{B_{i}, i}}{p_{i} G_{B_{i}, i}+\sum_{j \in U_{m}, j \notin B_{i}} p_{j} G_{B_{i}, j}+\sum_{k \in U_{f}, k \notin B_{i}} p_{k} G_{B_{i}, k}+\sigma_{B_{i}}^{2}} \\
& =\frac{\gamma_{i}(p)}{\gamma_{i}(p)+1}, \\
& \quad \forall i \in U, B_{i} \in B, \quad j \in U_{m}, k \in U_{f},(j, k) \notin B_{i} .
\end{aligned}
$$

Furthermore, the target SINR of user $i$ is denoted by $\widehat{\gamma}_{i}$, whereas the target effective SINR is represented by $\widehat{\Gamma}_{i}$. User $i$ is active if $\gamma_{i}(p) \geq \widehat{\gamma}_{i}$, or equivalently, if $\Gamma_{i}(p) \geq \widehat{\Gamma}_{i}$ for a given transmit power $0 \leq p_{i} \leq \bar{p}_{i}$. Alternatively, user $i$ is in an outage if $\gamma_{i}(p)<\widehat{\gamma}_{i}$, or equivalently, if $\Gamma_{i}(p)<\widehat{\Gamma}_{i}$ for a given transmit power $0 \leq p_{i} \leq \bar{p}_{i}$. The set of active users is denoted by $A=\left\{i \in U \mid \gamma_{i}(p) \geq \widehat{\Gamma}_{i}\right\}$ while the set of users in an outage is represented by $O(p)=\left\{i \in U \mid \Gamma_{i}(p)<\widehat{\Gamma}_{i}\right\}$. The sets of active MUEs and active FUEs are denoted by $A^{m}(p)=A(p) \cap U_{m}$ and $A^{f}(p)=A(p) \cap U_{f}$ while the sets of MUEs and FUEs in an outage are given by $O^{m}(p)=O(p) \cap U_{m}$ and set $O^{f}(p)=O(p) \cap U_{f}$. Now we can define the outage ratio of MUEs and FUEs as the ratio of the cardinality of sets $O^{m}$ and $O^{f}$ to the sets $U_{m}$ and $U_{f}$, respectively:

$$
\begin{aligned}
& \widehat{O^{m}}(p)=\frac{\left|O^{m}\right|}{\left|U_{m}\right|}, \\
& \widehat{O^{f}}(p)=\frac{\left|O^{f}\right|}{\left|U_{f}\right|} .
\end{aligned}
$$

In LTE-A, the uplink power for user $i$ in a given subframe is allocated through the FPC method [5]; that is,

$$
p_{i}(t+1)=\min \left\{\bar{p}_{i},\left(10 \log _{10}(M)+P_{o}+\propto \mathrm{PL}_{\mathrm{DL}}\right)\right\} .
$$

In the above expression, $p_{i}(t)$ is the uplink power for user $i$ in a given subframe and $\mathrm{PL}_{\mathrm{DL}}$ is the downlink path loss as estimated by the user. The $\bar{p}_{i}$ term denotes the maximum transmit power, $M$ indicates the instantaneous bandwidth measured in terms of physical resource blocks (PRBs), and $\propto$ is the partial path loss compensation factor that takes a value less than one. The parameter $P_{o}(t)$ is the desired target received power that takes the target SINR and instantaneous interference level into account and thus varies with time:

$$
P_{o}=f\left(p_{i}(t)\right)=\zeta_{i}(p) \widehat{\Gamma}_{i},
$$

where $\zeta_{i}(p)$ is the effective interference caused to user $i$ and $\widehat{\Gamma}_{i}$ is the effective target SINR of user $i$. The uplink power allocation in expression (7) mainly depends on parameter $P_{o}$. The power update equation can be rewritten as $P_{o}=$ $f\left(p_{i}(t)\right)=\left(\widehat{\gamma}_{i} / \gamma_{i}(p)\right) p_{i}(t)=\left(\widehat{\Gamma}_{i} / \Gamma_{i}(p)\right) p_{i}(t)$ in terms of the SINR and effective SINR. Though the power update function for the conventional power control method converges to a unique point, in HetNets, the users employing FPC stringently track their target SINR because the FPC method is conventionally designed for homogeneous networks and an interference scenario in HetNets is more severe as compared to homogeneous networks. Consequently, there always exist a few users that transmit at their maximum power, causing severe intercell and intertier interference which results in a high outage ratio.

3.2. Problem Statement. To minimize the outage ratio of FUEs subject to the zero-outage ratio of MUEs, the uplink power of all users must be dynamically controlled in the following conditions.

(i) The instantaneous effective SINR of all MUEs must be above their target SINR; that is, $\Gamma_{i}(p) \geq \widehat{\Gamma}_{i} \forall i \in U_{m}$.

(ii) The effective received interference level at the eNB must be below a given threshold; that is, $\zeta_{i}(p) \leq \bar{\zeta}_{i}$. If the received interference at the serving eNB is above the threshold level, the FUEs should be gradually removed.

\section{Interference-Aware Power Control}

In this section, first we discuss the total interference received at the eNB that will be used in our power control algorithms. Later in this section, we propose two interference-aware uplink power control algorithms for heterogeneous networks, keeping the constraint where the outage ratio for MUEs is zero with the minimum outage ratio for FUEs. In our proposed algorithms we impose a new limit on the transmit power of FUEs based on the total received interference at the eNB and the terminal's PHR in order to keep the interference caused by FUEs below a given threshold.

4.1. Total Received Interference at eNB. The total received interference at the serving eNB can be calculated by considering the interference from FUEs located in the serving macrocell region and MUEs from the neighboring cell. Thus, the total interference received at the serving eNB in a subframe is formally defined and obtained as

$$
\begin{aligned}
I_{i}(p)=\sum_{j \in U_{m}, j \notin B_{i}} p_{j} G_{B_{i}, j}+\sum_{k \in U_{f}, k \notin B_{i}} p_{k} G_{B_{i}, k}, \\
\quad \forall B_{i} \in B, \quad j \in U_{m}, k \in U_{f},(j, k) \notin B_{i} .
\end{aligned}
$$


The effective interference as can be seen from MUEs is formally defined and given by

$$
\begin{aligned}
& \zeta_{i}=\frac{I_{i}(p)}{G_{B_{i}, i}}=\frac{\sum_{j \in U_{m}, j \notin B_{i}} p_{j} G_{B_{i}, j}+\sum_{k \in U_{f}, k \notin B_{i}} p_{k} G_{B_{i}, k}}{G_{B_{i}, i}}, \\
& \forall i \in U, B_{i} \in B, j \in U_{m}, k \in U_{f},(j, k) \notin B_{i} .
\end{aligned}
$$

To ensure a zero-outage ratio for MUEs, the total received interference at each eNB must be below a given threshold. Given the total received interference at the eNB, $x \in B_{m}$, that is, $I_{i}(p)$, the effective target SINR of user $i \in U_{m}$ is reachable if and only if $0 \leq\left(\widehat{\Gamma}_{i} / G_{B_{i} i}\right)\left(\zeta_{i}\right) \leq \bar{p}_{i}$. The maximum value of the total effective received interference that can be tolerated by all MUEs in the serving eNB is denoted by $\bar{\zeta}_{i}$ and can be obtained as follows:

$$
\begin{aligned}
\bar{\zeta}_{i} & =\max \left\{\zeta_{i} \mid 0 \leq \frac{\widehat{\Gamma}_{i}}{G_{B_{i}, i}}\left(\zeta_{i}\right) \leq \bar{p}_{i}\right\} \\
& =\min \left\{\frac{\bar{p}_{i} G_{B_{i}, i}}{\widehat{\Gamma}_{i}}\right\}-\left(\zeta_{i}+\sigma_{B_{i}}^{2}\right) .
\end{aligned}
$$

If the transmit power vector $\mathbf{p}$ satisfies the SINR requirements of all MUEs in the associated eNB, then $\zeta_{i}(p) \leq \bar{\zeta}_{i}$, or equivalently, $\max \left\{\zeta_{i}(p) / \bar{\zeta}_{i}\right\} \leq 1$.

4.2. Power Headroom Report. The power headroom report (PHR) is the measure of the difference between the user maximum transmit power and the estimated transmit power that would have been used if there was no upper limit on transmit power. The PHR for a specific subframe as defined in [23] can be obtained as

$$
\mathrm{PHR}=\bar{p}_{i}-\left(10 \log _{10}(M)+P_{o}+\propto \mathrm{PL}_{\mathrm{DL}}\right) .
$$

The PHR can either be positive or negative. A positive PHR indicates that the actual transmit power was lower than the maximum terminal transmit power. However, a negative PHR indicates that the transmit power was limited by the maximum power constraint, that is, $\bar{p}_{i}$. To calculate the transmit power for the next subframe based on the PHR, we define two terms, the instantaneous PHR difference and average PHR difference denoted by $\lambda$ and $\bar{\lambda}$, respectively, which can be obtained as

$$
\begin{aligned}
& \lambda=\mathrm{PHR}_{i}-\mathrm{PHR}_{i-1}, \\
& \bar{\lambda}=\frac{1}{Z} \sum_{i=1}^{Z} \mathrm{PHR}_{i},
\end{aligned}
$$

where $\mathrm{PHR}_{i}$ denotes the PHR of the current subframe while $\mathrm{PHR}_{i-1}$ denotes the PHR of the previous subframe and $\bar{\lambda}$ represents the average of PHR difference over $Z$ previous optimum values of PHR difference readings. The PHR difference can be used as an indicator of the channel condition. A positive value of an instantaneous PHR difference, that is, $\lambda>0$, indicates that the channel gain at a specific subframe is increased as compared to the previous subframe, while a negative value of an instantaneous PHR difference, that is, $\lambda<0$, shows that the channel gain is decreased at the given subframe in comparison to the previous subframe. To minimize the outage ratio of FUEs subject to the zero-outage ratio for MUEs, the received effective interference at the serving eNB should be reduced by dynamically updating the transmit power of all users $i \in U$ in proportion to the increase or decrease in the channel gain that can be estimated by the PHR factor denoted by $\hat{\lambda}$ and is given by $\hat{\lambda}=\bar{\lambda} /(\bar{\lambda}+\lambda)$.

4.3. Interference-Aware Uplink Power Control Algorithm. The proposed transmit power control algorithm takes the total received interference at the serving eNB that can be tolerated by all associated MUEs while allocating power to the user terminal. The power update equation of the proposed power control method is given by

$$
p_{i}(t+1)= \begin{cases}\min \left\{\bar{p}_{i}, \frac{\widehat{\Gamma}_{i}}{\Gamma_{i}(p)} p_{i}(t)\right\} & \forall i \in U_{m}, \\ \min \left\{\bar{p}_{i}, \delta(t) \frac{\widehat{\Gamma}_{i}}{\Gamma_{i}(p)} p_{i}(t)\right\} & \forall i \in U_{f},\end{cases}
$$

where $\delta(t)$ is defined as a power controlling factor and can be obtained as

$$
\delta(t)= \begin{cases}\frac{\bar{\zeta}_{i}}{\bar{\zeta}_{i}(p)} & \text { for }\left\{\frac{\zeta_{i}(p)}{\bar{\zeta}_{i}}\right\}>1, \\ 1 & \text { for }\left\{\frac{\zeta_{i}(p)}{\bar{\zeta}_{i}}\right\} \leq 1\end{cases}
$$

where $\bar{\zeta}_{i}$ is the maximum tolerable effective interference by all MUEs at the eNB and $\zeta_{i}(p)$ is the instantaneous effective interference at the eNB. In IA-TPC, all MUEs transmit power according to the conventional FPC method. However, all the FUEs transmit power according to the conventional FPC method as long as the total received interference at the serving eNB is less than the given threshold, $\zeta_{i}(p) \leq$ $\bar{\zeta}_{i}$. Instead, if the total effective received interference at the serving eNB is greater than the given threshold, $\zeta_{i}(p)>\bar{\zeta}_{i}$, the transmit power of all FUEs is limited according to the controlling factor $\delta(t)$. The controlling factor $\delta(t)$ takes the total tolerable received interference at the serving eNB into account while allocating power to the FUEs. The proposed power update equation certainly reduces to the conventional FPC equation as long as the total received interference is below the given threshold. Further, the IA-TPC algorithm can be considered the same as the closed-loop power control method with two power updating commands. First, ratio $\widehat{\Gamma}_{i} / \Gamma_{i}(p)$ controls the transmit power based on the effective SINR conditions that are unicast to all users by its own receiver. Second, the controlling factor $\delta(t)$ can be viewed as the power updating command based on the interference situation and is multicast to FUEs by their respective feNB.

Moreover, the proposed IA-TPC method uses a dynamic approach for setting the interference threshold. When there are many MUEs with high target SINR requirements and/or 
with poor channel conditions, the total tolerable received interference threshold is set to a very small value. Alternately, when there is a small number of MUEs with low SINR requirements and/or the channel condition is good, the total tolerable received interference threshold can be set to a relatively large value.

4.4. PHR-Based IA-TPC. The formally proposed IA-TPC algorithm guarantees a zero-outage ratio for the MUEs, yet the transmit power equation with minimal outage for FUEs is preferred. According to (15), if $\left\{\zeta_{i}(p) / \bar{\zeta}_{i}\right\}>1$, all FUEs reduce their transmit power in proportion to $\min \left\{\bar{p}_{i} G_{B_{i}, i} / \widehat{\Gamma}_{i}\right\}-\left(\zeta_{i}+\right.$ $\sigma_{B_{i}}^{2}$ ) in order to minimize the interference caused by FUEs to MUEs. However, it is more effective if the FUEs that cause interference to the serving $\mathrm{eNB}$ reduce their transmit power in proportion to the interference caused by the increase in channel gain. The effective interference $\zeta_{i}=I_{i}(p) / G_{B_{i}, i}$ at the serving eNB can certainly be dropped drastically given the condition that all terminals update their transmit power in proportion to the PHR factor, which is the eventual measure of the increase or decrease in channel gain. Here, we propose a novel PHR-based IA-TPC algorithm in which all users (including MUEs) update their transmit power in proportion to the PHR factor. The PHR-based IA-TPC algorithm has the following power update function:

$$
\begin{aligned}
& p_{i}(t+1) \\
& = \begin{cases}\min \left\{\bar{p}_{i}, \hat{\lambda}(t) \frac{\widehat{\Gamma}_{i}}{\Gamma_{i}(p)} p_{i}(t)\right\} & \forall i \in U_{m}, \\
\min \left\{\bar{p}_{i}, \hat{\lambda}(t) \delta(t) \frac{\widehat{\Gamma}_{i}}{\Gamma_{i}(p)} p_{i}(t)\right\} & \forall i \in U_{f},\end{cases}
\end{aligned}
$$

where the PHR factor $(\widehat{\lambda}(t)=\bar{\lambda} /(\bar{\lambda}+\lambda))$ is the new limit on the uplink transmit power that considers the instantaneous changes in the channel condition, calculated from the PHR, while updating the transmit power of the user terminals. Indeed, the proposed PHR-based IA-TPC power update equation also reduces to the conventional FPC equation as long as the total received interference is below the given threshold and the channel condition remains constant. Further, the PHR-based IA-TPC algorithm can be considered the same as the FPC method with three power updating commands. First, the ratio $\widehat{\Gamma}_{i} / \Gamma_{i}(p)$ controls the transmit power based on the effective SINR conditions that are unicast to all users by its own receiver. Second, the controlling factor $\delta(t)$ can be viewed as the power updating command based on the interference situation and is multicast to FUEs by their respective feNB. Third, the PHR factor $\hat{\lambda}(t)$ is the power adjusting factor that is based on the channel condition and is calculated locally at the user terminal from its PHR measurement.

\section{Discussion}

5.1. Signaling Overhead of the Proposed Algorithm. In the proposed IA-TPC algorithm, besides the data that each user terminal requires in order to update their transmit power using the FPC, each FUE needs to know the ratio of the maximum tolerable interference to the instantaneous interference received at the eNB, $\bar{\zeta}_{i} / \zeta_{i}(p)$, which is obtained from the eNB. The value of the ratio $\bar{\zeta}_{i} / \zeta_{i}(p)$ is multicast by the eNB to all associated feNBs which in turn forward the obtained value to all FUEs connected in the CSG. From a practical perspective, the feedback information can be quantized from an appropriate number of bits. Then, these bits can be communicated to the feNB by the eNB. Then the feNB sends this information to its associated FUEs. Here, it should be observed that there is no such type of $\mathrm{X} 2$ interface between the $\mathrm{eNB}$ and $\mathrm{feNB}$ in practice for information exchange. Hence, the eNB should depend on the physical uplink control channel (PUCCH) to provide the information to the feNB. Therefore, in the proposed IA-TPC in comparison with FPC, each eNB needs to provide extra signaling overhead of $\bar{\zeta}_{i} / \zeta_{i}(p)$ to the feNB at each iteration. However, in PHR-based IA-TPC the PHR factor $\hat{\lambda}(t)=\bar{\lambda} /(\bar{\lambda}+$ $\lambda)$ is calculated locally at the user terminal and, hence, no extra signaling overhead is required as compared to the IATPC algorithm.

5.2. Valid Power Vector Existence. In this subsection we demonstrate that, for the proposed IA-TPC and PHR-based IA-TPC algorithms, there always exists at least one valid power vector that ensures zero-outage for MUEs given any effective interference at the eNB. Let us denote a valid power vector by $\overline{\overline{\mathbf{p}}}(\gamma)$, obtained from the IA-TPC/PHR-based IATPC power update equation at a given SINR vector, $\gamma=$ $\left[\gamma^{m}, \gamma^{f}\right]$. At a given target SINR vector $\hat{\gamma}=\left[\widehat{\gamma}^{m}, \widehat{\gamma}^{f}\right]$, the corresponding valid power vector is represented by $\overline{\overline{\mathbf{p}}}(\widehat{\gamma})$. The maximum effective interference received at the eNB that can be tolerated by all MUEs associated with the serving eNB is denoted by $\bar{\zeta}_{i}$, whereas the instantaneous effective interference for the given valid power vector is denoted by $\zeta_{i}(\overline{\overline{\mathbf{p}}}(\widehat{\gamma}))$. At the given maximum tolerable effective interference $\bar{\zeta}_{i}$, instantaneous effective interference $\zeta_{i}(\overline{\overline{\mathbf{p}}}(\widehat{\gamma}))$, and valid power vector $\overline{\overline{\mathbf{p}}}(\widehat{\gamma})$, the following two possibilities can occur.

Case $1\left(\left\{\zeta_{i}(\overline{\overline{\mathbf{p}}}(\widehat{\gamma})) / \bar{\zeta}_{i}\right\} \geq 1\right)$. If the instantaneous effective interference is equal to or higher than the maximum tolerable effective interference, then Case 1 infers that there may exists one or more MUEs which are in outage if the power is updated with the FPC algorithm. For the given scenario, we have to show that the power vector $\mathbf{p}(\widehat{\gamma})$ updated with the proposed algorithm is a valid power vector $\overline{\overline{\mathbf{p}}}(\widehat{\gamma})$ and that the following equalities and inequalities hold for Case 1 :

$$
\begin{aligned}
0 & \leq p_{i}(\gamma) \leq \bar{p}_{i}(\gamma), \\
\gamma^{m}(\overline{\overline{\mathbf{p}}}) & =\widehat{\gamma}^{m}, \\
\gamma^{f}(\overline{\overline{\mathbf{p}}}) & \leq \hat{\gamma}^{f} .
\end{aligned}
$$

Proof. Let us define an MUE-protected target SINR vector by $\tilde{\gamma}=\left[\widehat{\gamma}^{m}, 0\right]$, where $\widetilde{\gamma}<\widehat{\gamma}$. In addition, we also know that the target SINR for all MUEs is achievable in the absence of 
FUEs (i.e., $\widehat{\gamma}^{f}=0$ ). Further, it has been previously shown that if the transmit power vector $\mathbf{p}$ satisfies the SINR requirements of all MUEs in the associated eNB, then $\zeta_{i}(p) \leq \bar{\zeta}_{i}$. As $\tilde{\gamma}<\widehat{\gamma}$, this implies that $\zeta_{i}(\mathbf{p}(\widetilde{\gamma})) \leq \zeta_{i}(\mathbf{p}(\widehat{\gamma})) \forall$ eNBs. Thus, if $\left\{\zeta_{i}(\overline{\overline{\mathbf{p}}}(\widehat{\gamma})) / \bar{\zeta}_{i}\right\} \geq 1$, or equivalently, if $\zeta_{i}(\overline{\overline{\mathbf{p}}}(\widehat{\gamma})) \geq \bar{\zeta}_{i}$, in the IATPC algorithm, we have $\zeta_{i}(\overline{\overline{\mathbf{p}}}(\widehat{\gamma})) \geq \bar{\zeta}_{i} \geq \zeta_{i}(\overline{\overline{\mathbf{p}}}(\tilde{\gamma}))$. Therefore, this shows that there exists an SINR vector where $\tilde{\gamma} \leq \gamma \leq \hat{\gamma}(\gamma$ corresponds to $\left.\bar{\zeta}_{i}\right)$. Furthermore, the controlling factor $\delta(t)$ in the IA-TPC algorithm is a limiting factor and is less than one for the given case; there exists a valid transmit power vector $\overline{\overline{\mathbf{p}}}(\widehat{\gamma})$ for which $0 \leq p_{i}(\gamma) \leq \bar{p}_{i}(\gamma)$. Moreover, the inequality $\tilde{\gamma} \leq \gamma \leq \hat{\gamma}$ proves that $\gamma^{m}(\overline{\overline{\mathbf{p}}})=\widehat{\gamma}^{m}$ and $\gamma^{f}(\overline{\overline{\mathbf{p}}}) \leq \widehat{\gamma}^{f}$. Similarly, for PHR-based IA-TPC, the same proof holds as it follows the same set of rules as implied by the IA-TPC-based algorithm while updating the power vector.

Case $2\left(\left\{\zeta_{i}(\overline{\overline{\mathbf{p}}}(\widehat{\gamma})) / \bar{\zeta}_{i}\right\}<1\right)$. If the instantaneous effective interference is less than the maximum tolerable effective interference, then Case 2 infers that no MUE is in outage if the power is updated with the FPC algorithm. For this scenario, we have to show that the power vector $\mathbf{p}(\widehat{\gamma})$, updated with the proposed algorithm, is a valid power vector $\overline{\overline{\mathbf{p}}}(\widehat{\gamma})$ and that the following equalities and inequalities hold for Case 2:

$$
\begin{aligned}
0 & \leq p_{i}(\gamma) \leq \bar{p}_{i}(\gamma), \\
\gamma^{m}(\overline{\overline{\mathbf{p}}}) & \geq \widehat{\gamma}^{m}, \\
\gamma^{f}(\overline{\overline{\mathbf{p}}}) & \geq \widehat{\gamma}^{f} .
\end{aligned}
$$

Proof. If $\left\{\zeta_{i}(\overline{\overline{\mathbf{p}}}(\widehat{\gamma})) / \bar{\zeta}_{i}\right\}<1$, or in other words, $\zeta_{i}(\overline{\overline{\mathbf{p}}}(\widehat{\gamma}))<\bar{\zeta}_{i} \forall$ eNBs, then it is concluded that the target SINR is achievable by all the users (i.e., $\gamma>\widehat{\gamma} \forall i \in U$ ). Correspondingly, if the target SINR is achieved, it can be concluded that $\gamma^{m}(\overline{\overline{\mathbf{p}}}) \geq$ $\widehat{\gamma}^{m}$ and $\gamma^{f}(\overline{\overline{\mathbf{p}}}) \geq \widehat{\gamma}^{f}$, because, otherwise, if $\gamma \leq \hat{\gamma}$, then $\zeta_{i}(\mathbf{p}(\gamma)) \leq \zeta_{i}(\mathbf{p}(\widehat{\gamma}))$. Additionally, for any two SINRs $\gamma_{1}$ and $\gamma_{2}$ and their corresponding power vectors $\mathbf{p}_{\mathbf{1}}$ and $\mathbf{p}_{\mathbf{2}}$, if $\gamma_{1}<\gamma_{2}$ then $\mathbf{p}_{1}<\underline{\mathbf{p}}_{2}$ and ultimately $\zeta_{i}\left(\mathbf{p}_{\mathbf{1}}\right) \leq \zeta_{i}\left(\mathbf{p}_{\mathbf{2}}\right)$. This contradicts $(\overline{\overline{\mathbf{p}}}(\widehat{\gamma}))<\bar{\zeta}_{i}$. Thus, it is proved that $\gamma^{m}(\overline{\overline{\mathbf{p}}}) \geq \widehat{\gamma}^{m}$ and $\gamma^{f}(\overline{\overline{\mathbf{p}}}) \geq \widehat{\gamma}^{f}$. Further, for the given scenario, the value of the controlling factor $\delta(t)$ in the IA-TPC algorithm is considered to be one and the power is limited by the effective SINR ratio $\widehat{\Gamma}_{i} / \Gamma_{i}(p)$. Moreover, we know that, in the given scenario, $\gamma>\widehat{\gamma}$, and correspondingly $\Gamma_{i}((\overline{\overline{\mathbf{p}}})) \geq \widehat{\Gamma}_{i}$; thus, there exists a valid transmit power vector $\overline{\overline{\mathbf{p}}}(\widehat{\gamma})$ for which $0 \leq p_{i}(\gamma) \leq \bar{p}_{i}(\gamma)$. Similarly, the same proof is valid for PHR-based IA-TPC, as it follows the same set of rules as implied by the IA-TPC-based algorithm while updating the power vector.

5.3. Outage Ratio. In this section we show that the proposed algorithms ensure zero-outage for the MUEs at any valid power vector obtained with the power update function of IATPC and PHR-based IA-TPC: that is, $O^{m}(\overline{\overline{\mathbf{p}}})=0$. To prove zero-outage for MUEs at the valid power vector $\overline{\overline{\mathbf{p}}}(\gamma)$, that is, $O^{m}(\overline{\overline{\mathbf{p}}})=0$, the following equalities and inequalities hold:

$$
\begin{aligned}
0 & \leq \overline{\overline{\mathbf{p}}}_{\mathbf{i}}(\gamma) \leq \bar{p}_{i}(\gamma), \\
\gamma^{m}(\overline{\overline{\mathbf{p}}}) & \geq \hat{\gamma}^{m} .
\end{aligned}
$$

Proof. From our discussion in the previous subsection, we know that, for any valid power vector $\zeta_{i}(\mathbf{p}(\widehat{\gamma})) \leq \bar{\zeta}_{i}, \bar{\zeta}_{i}$ is the maximum effective interference that can be tolerated by all MUEs in the serving eNB. If $\zeta_{i}(\mathbf{p}(\widehat{\gamma}))<\bar{\zeta}_{i} \forall$ eNBs, then this implies that $\Gamma_{i}((\overline{\overline{\mathbf{p}}})) \geq \widehat{\Gamma}_{i}$; therefore, the magnitude of the ratio $\widehat{\Gamma}_{i} / \Gamma_{i}(\overline{\overline{\mathbf{p}}}) \leq 1$, and hence a valid transmit power vector is given by $\overline{\overline{\mathbf{p}}}_{\mathbf{i}}(\gamma) \leq \bar{p}_{i}(\gamma)$. Further, $\zeta_{i}(\mathbf{p}(\widehat{\gamma})) \leq \bar{\zeta}_{i}$ indicates that the target SINR is achievable by all of the users (i.e., $\gamma>\widehat{\gamma} \forall i \in$ $U)$. Correspondingly, if the target SINR is achieved it can be concluded that $\gamma^{m}(\overline{\overline{\mathbf{p}}}) \geq \widehat{\gamma}^{m}$. This proves that, for all MUEs, $\gamma^{m}(\overline{\overline{\mathbf{p}}}) \geq \widehat{\gamma}^{m}$, or in other words, $O^{m}(\overline{\overline{\mathbf{p}}})=0$.

\section{Performance Evaluation}

In this section, we evaluate the performances of the proposed algorithms based on system-level simulations. We used the HetNet deployment as given in the 3GPP LTE specifications in our simulations. The simulations are carried out using MATLAB. The in-depth details of the simulation model, evaluation criterion, and simulation results are discussed in the following subsections.

6.1. Simulation Model and Evaluation Criterion. The simulation model is developed according to the standard LTE network with an ultra-dense HetNet environment as shown in Figure 1. The model consists of 7 eNBs where CSG-based femtocells are randomly deployed that reuse the same carrier frequency to create a HetNet environment. The service area of each eNB and feNB is considered to have a $500 \mathrm{~m}$ intersite distance and $20 \mathrm{~m}$ radius, respectively. Each MUE/FUE is equipped with a single antenna while the user speed is assumed to be $3 \mathrm{~km} / \mathrm{h}$. We have assumed full buffer traffic in our simulations. The channel is considered to follow the Claussen model [24], which incorporates path loss and shadowing in urban environments. Moreover, in the proposed algorithms we have set a $100 \mathrm{~ms}$ slot length (i.e., after every 10 frames) for the power update function. The transmission time interval (TTI) for each subframe is $1 \mathrm{~ms}$ as given in the LTE specifications. However, this time duration is too small for the purpose of power updates because the channel conditions and interference situation changes much slower as compared to the TTI. Other simulation parameters are set in accordance with the LTE standard and are summarized in Table 1.

In our simulations, we evaluate the performances of the proposed algorithms summarized in (15) and (17), termed IA-TPC and PHR, in comparison to the conventional FPC algorithm specified in (7). In the simulations, we used a cumulative distribution function (CDF) of the MUE and FUE average throughput and a CDF of the received interference at the eNB in order to compare the three algorithms. Further, we compare the average throughput of MUEs and FUEs 
TABLE 1: Simulation parameters.

\begin{tabular}{lc}
\hline Parameter & Assumed value \\
\hline Cell layout & Hexagonal grid, \\
Number of sites & 3 cells per eNB \\
Service area & eNB $(=21$ cells) \\
Carrier frequency & feNB $=20$ m radius \\
Bandwidth & $1.76 \mathrm{GHz}$ \\
MUEs distribution & $10 \mathrm{MHz}(50 \mathrm{PRBs})$ \\
FUE distribution & 100 users randomly distributed \\
Number of feNBs & (average 15 per eNB) \\
per eNB & 3 FUEs per feNB \\
Channel & 20 feNBs randomly distributed \\
& Path loss (urban) \\
Maximum & Shadowing (Claussen model $[21])$ \\
transmit power & eNB: $46 \mathrm{dBm}$ \\
feNB: $20 \mathrm{dBm}$ \\
Transmit power \\
update interval \\
Traffic model \\
Interference
\end{tabular}

relative to the increasing interference situation. In addition, the transmit power allocated to MUEs and FUEs is compared based on the proposed and conventional power update algorithms. Finally, the outage ratio, defined in (5) and (6), is used to evaluate the outage performances of the proposed algorithms.

6.2. Simulation Results. For the previously discussed model, the CDFs of the average throughput of MUEs and FUEs are simulated for the three algorithms, IA-TPC, PHR, and FPC, and the results are plotted in Figures 2 and 3. From the throughput CDF curves, the following information can be deduced:

(i) First, the MUE throughput of the PHR algorithm significantly outperforms the IA-TPC and FPC algorithms as can be seen from Figure 2. This is mainly because the PHR algorithm updates the transmit power in accordance with instantaneous channel conditions, thereby reducing the interference results and increasing the average throughput. Additionally, the IA-TPC algorithm also outperforms the conventional FPC algorithm for MUEs. Although for the MUE case the IA-TPC is reduced to FPC due to less interference

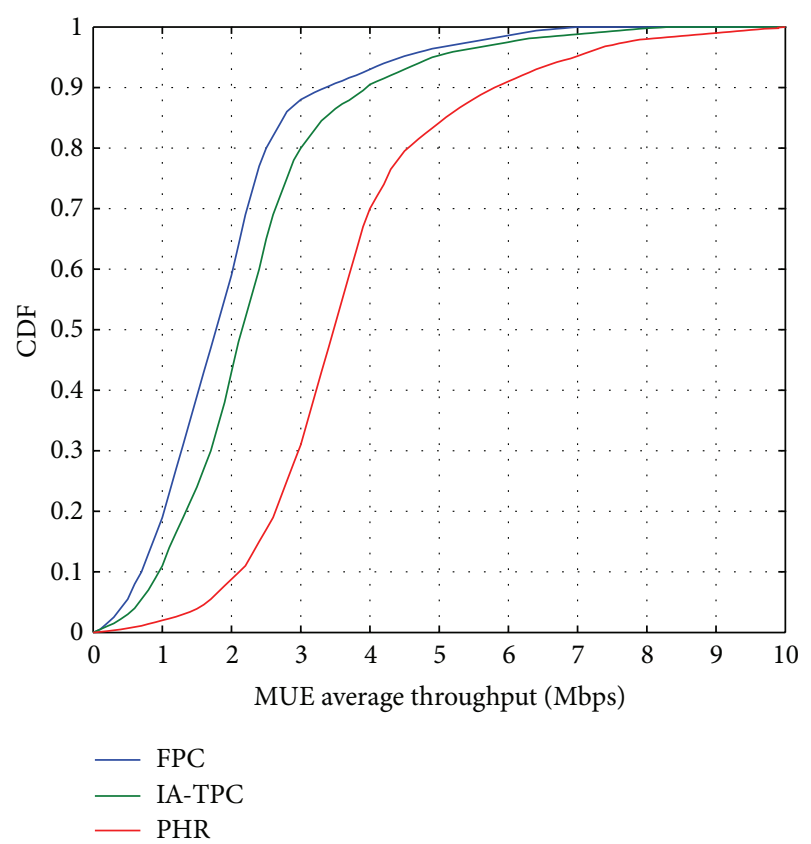

FIgure 2: Average throughput CDF of MUE in HetNet environment.

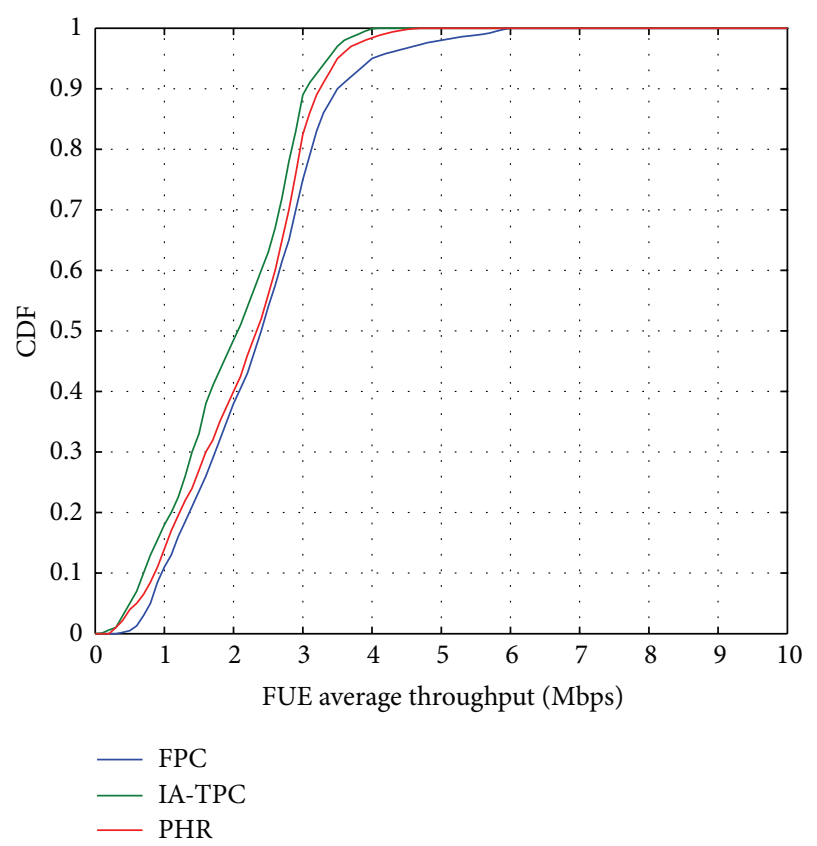

FIGURE 3: Average throughput CDF of FUE in HetNet environment.

from FUEs, the average throughput of the IA-TPC algorithm is more than that of the conventional algorithm.

(ii) However, for FUE throughput, as shown in Figure 3, the conventional FPC performs slightly better than the proposed algorithms. To understand this scenario, recall that, in the proposed algorithm, when the 


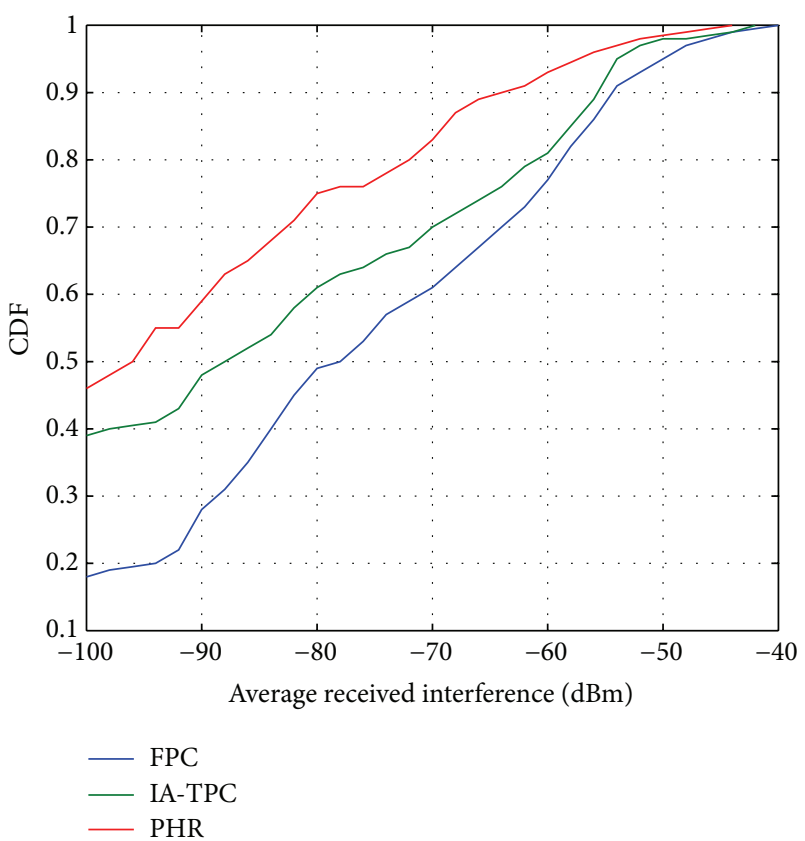

FIgURE 4: Received interference at eNB in HetNet environment.

received interference is increased beyond the threshold, some FUEs may be gradually removed from the network, resulting in decreased throughput.

(iii) Secondly, for the cell edge users (i.e., below 5\% user throughput CDF in the graph), the IA-TPC and PHR give almost the same performance, while FPC gives a better performance, specifically in the FUE case. The reason for this is that along the cell edge, the instantaneous interference becomes larger than the threshold interference resulting in outage for FUEs. Hence, the throughput of FUEs is decreased whereas that of the MUEs is protected.

Figure 4 shows the $\mathrm{CDF}$ of the average received interference at the eNB. For average users (i.e., at $50 \%$ of the CDF), the curves show that the received interference for the PHR and IA-TPC algorithms is almost $17 \%$ and $8 \%$ less than the conventional FPC algorithm. This is because the proposed algorithms consider the received interference at the eNB while allocating transmit power to the terminals. The PHR algorithm outperforms the FPC and IA-TPC algorithm due to the aforementioned reason.

Next, we show the average throughput of an MUE/FUE against the varying interference. In our simulations, we set the threshold interference equal to $5 \mathrm{~dB}$. In Figure 5, the average throughput of an MUE is plotted against the interference. At the threshold value (i.e., $5 \mathrm{~dB}$ ), the MUE average throughput is rapidly decreased in the case of the FPC algorithm, while, for the proposed algorithms, the rate of decrease in average throughput for the MUE is slow. In order to understand this scenario, recall that when the interference level is increased above the threshold, the proposed algorithms drastically decrease the transmit power of FUEs (FUE may go into outage), thereby maintaining the MUE throughput. On the

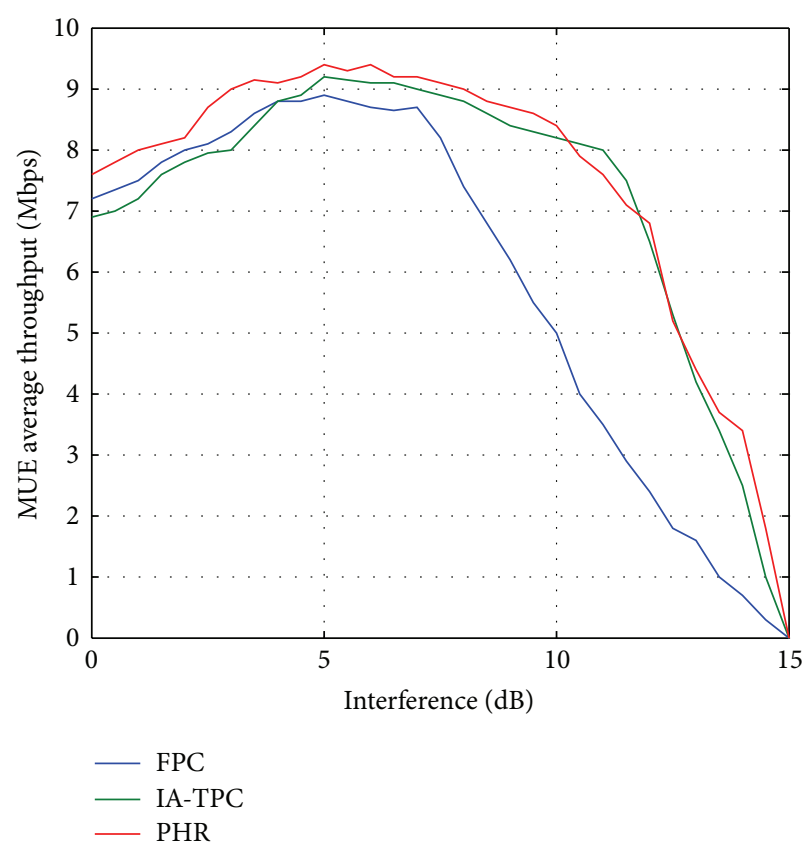

FIGURE 5: MUE throughput versus received interference at eNB (at $5 \mathrm{~dB}$ threshold).

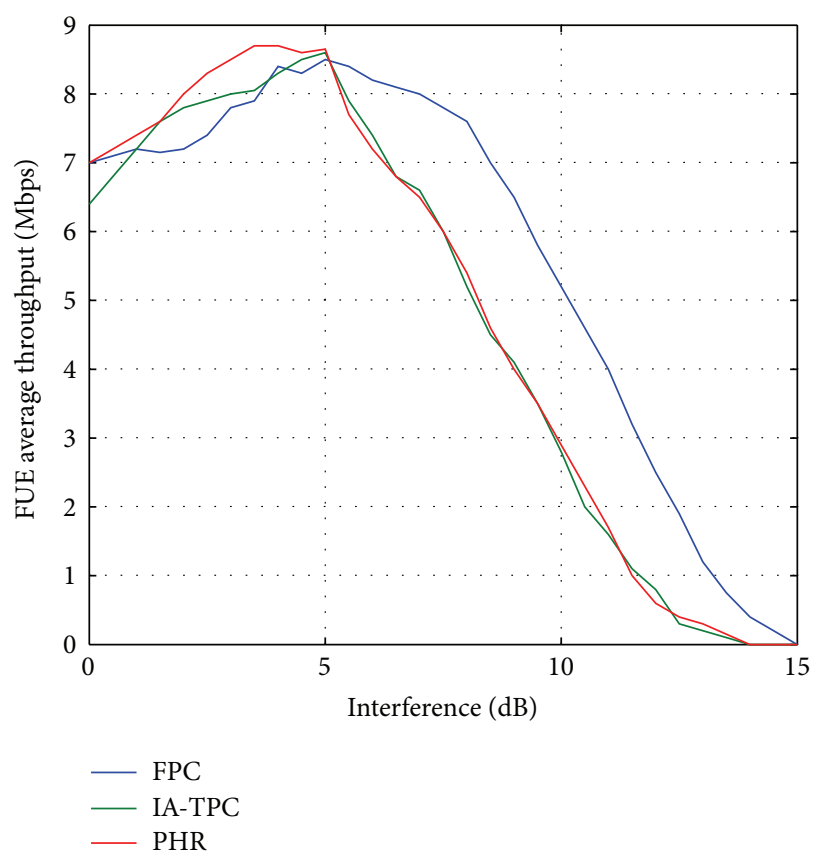

FIGURE 6: FUE throughput versus received interference at eNB (at $5 \mathrm{~dB}$ threshold).

other hand, as shown in Figure 6, when the interference increases above the threshold level, the average throughput of FUEs decreases rapidly in the case of the proposed algorithms as compared to the FPC algorithm. The reason for this is that the transmit power of the FUEs is significantly decreased by the proposed algorithms (FUEs may go into outage) in order to protect the MUEs. 


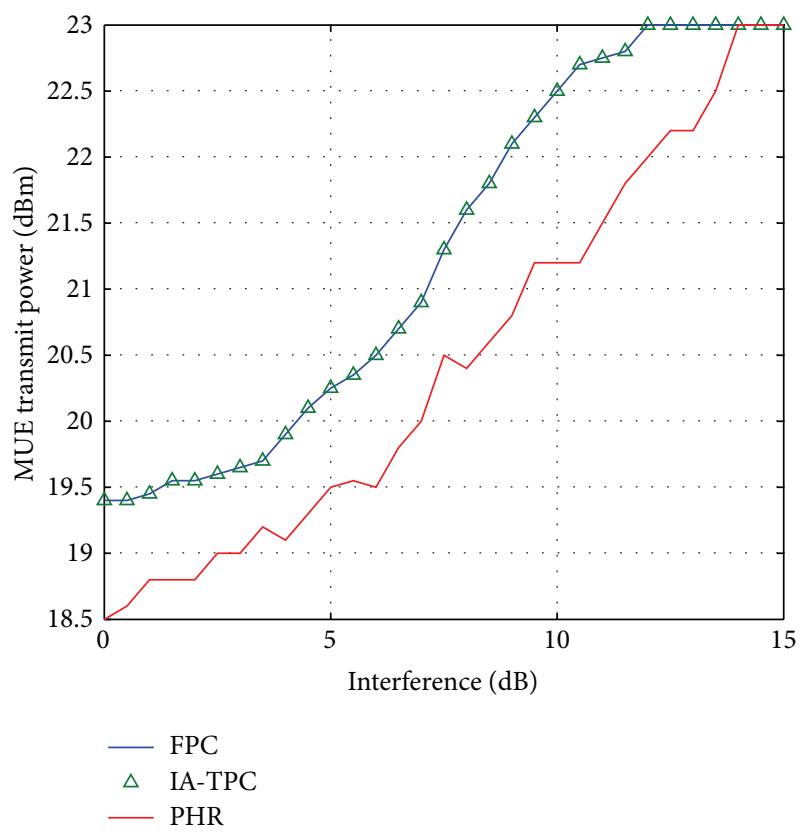

FIGURE 7: MUE transmit power versus received interference at eNB (at $5 \mathrm{~dB}$ threshold).

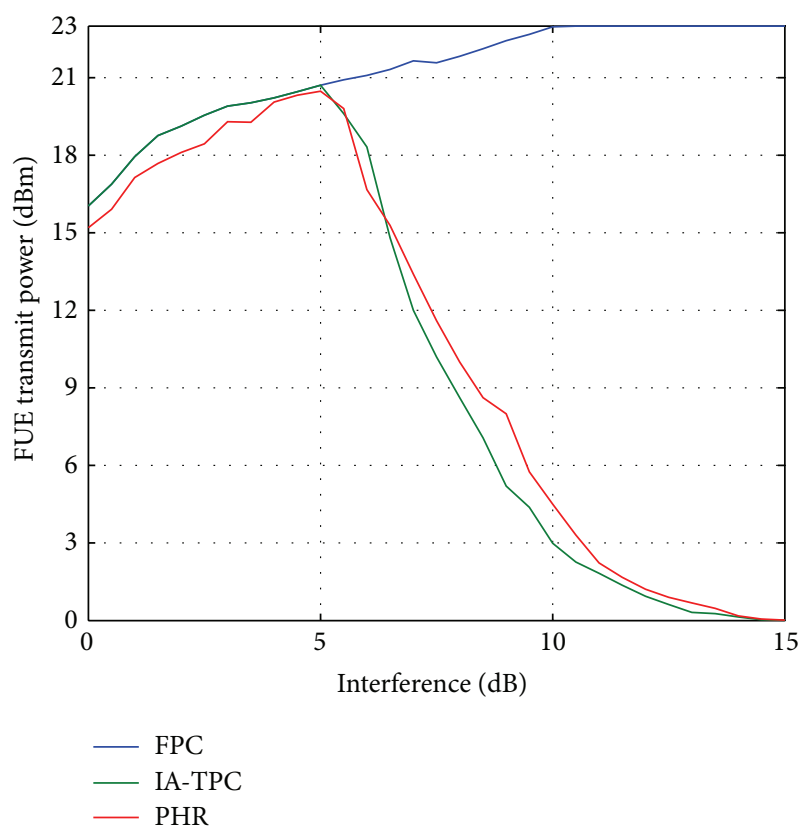

FIGURE 8: FUE transmit power versus received interference at eNB (at $5 \mathrm{~dB}$ threshold).

Further, Figures 7 and 8 show the allocated transmit power to the MUE and FUE at the varying interference levels. In the case of MUEs, as given in Figure 7, the transmit power is increased gradually with increased interference. The IA-TPC and FPC algorithms assign the same power to the user terminal while the PHR allots less power since it considers instantaneous changes in the channel condition while allocating power. Instead, the FUE transmit power

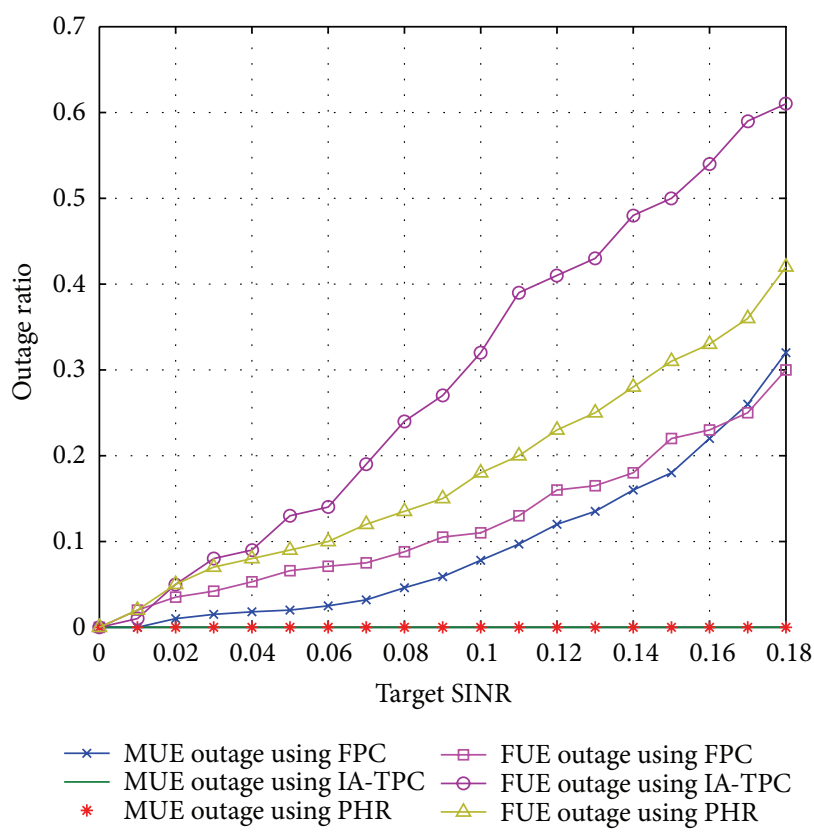

FIGURE 9: Outage performance versus target SINR.

is decreased drastically by the proposed algorithms when the interference level goes beyond the threshold level, as shown by the red and green curves in Figure 8 . However, the FPC algorithm allocates more transmit power to mitigate the interference. Hence, it can be anticipated that MUE protection is guaranteed at the cost of FUE outage.

Finally, in Figure 9 we describe the average outage ratio of an MUE and FUE versus the target SINR for the proposed and conventional algorithms. Note that, in the case of MUEs, both of the proposed algorithms outperform the conventional FPC by ensuring zero-outage for the MUE at the cost of increased outage for FUEs. As can be seen from the green and red star curves, the MUE outage is zero for the IA-TPC and PHR algorithms while the blue curve shows some outage ratio for the MUE. However, in the case of FUEs, the outage ratio for FPC is less than that of the PHR and IA-TPC. Moreover, the outage ratio of the FUE using the PHR is less than the outage ratio of the FUE using IA-TPC since in the PHR, the FUEs cause more interference (due to channel gain) to MUEs and accordingly reduce their transmit power more, thereby resulting in a lower outage ratio.

\section{Conclusion}

In this paper, we proposed interference-aware transmit power control (IA-TPC) and power headroom report-based IA-TPC algorithms for LTE-A heterogeneous networks with the aim of achieving zero-outage for the macrocells users. With the IA-TPC algorithm, we showed that the proposed algorithm not only ensures zero-outage for the macrocells users but also increases the macrocells users' average throughput, even above the received interference threshold level, at the cost of an increased outage ratio for femtocells users. Further, with the PHR-based IA-TPC algorithm, we showed that, along 
with the zero-outage for macrocells users, the outage ratio for femtocells users can also be reduced by properly updating the transmit power by taking the current channel condition into consideration. The performances of the proposed algorithms were evaluated using simulations in the MATLAB environment. The results show that the proposed algorithms perform significantly better than the conventional transmit power update method. In their future consideration, the authors' focus is on flexible physical resource blocks (PRBs) allocation based on the user's power capability and channel condition. The significance of flexible PRBs allocation is twofold: (i) efficient resource allocation and (ii) decreasing harmful interference, which will eventually result in throughput enhancements and a decrease in outage ratio.

\section{Competing Interests}

The authors declare that there is no conflict of interests regarding the publication of this paper.

\section{Acknowledgments}

This work was supported by Human Resource Program of Korea Institute of Energy Technology Evaluation and Planning (KETEP), Ministry of Trade, Industry \& Energy, Republic of Korea, under Grant no. 20154030200730.

\section{References}

[1] 3GPP, Evolved universal terrestrial radioaccess (E-UTRA); User equipment (UE) radio transmission and reception (release 12), 3GPP TS 36.101, 2014.

[2] M. Peng, C. Wang, J. Li, H. Xiang, and V. Lau, "Recent advances in underlay heterogeneous networks: interference control, resource allocation, and self-organization," IEEE Communications Surveys \& Tutorials, vol. 17, no. 2, pp. 700-729, 2015.

[3] Q. Zhang, T. Yang, Y. Zhang, and Z. Feng, "Fairness guaranteed joint CRE and eICIC scheme for capacity improvement in twotier heterogeneous networks," Electronics Letters, vol. 50, no. 24, pp. 1817-1819, 2014.

[4] A. Damnjanovic, J. Montojo, Y. Wei et al., "A survey on 3GPP heterogeneous networks," IEEE Wireless Communications, vol. 18 , no. 3, pp. 10-21, 2011.

[5] Z. Luo, R. Gilimyanov, H. Zhuang, and J. Zhang, "Networkwide optimization of uplink fractional power control in LTE networks," in Proceedings of the IEEE 82nd Vehicular Technology Conference (VTC Fall '15), pp. 1-5, Boston, Mass, USA, 2015.

[6] M. Boussif, C. Rosa, J. Wigard, and R. Müllner, "Load adaptive power control in LTE Uplink," in Proceedings of the European Wireless Conference (EW '10), pp. 288-293, Lucca, Italy, April 2010.

[7] C. U. Castellanos, F. D. Calabrese, K. I. Pedersen, and C. Rosa, "Uplink interference control in UTRAN LTE based on the overload indicator," in Proceedings of the IEEE 68th Vehicular Technology Conference (VTC-Fall '08), pp. 1-5, Calgary, Canada, September 2008.

[8] S. Deb and P. Monogioudis, "Learning-based uplink interference management in 4G LTE cellular systems," IEEE/ACM Transactions on Networking, vol. 23, no. 2, pp. 398-411, 2015.
[9] G. Giambene and V. A. Le, "Performance evaluation of different fractional frequency reuse schemes for LTE," in Proceedings of the Euro Med Telco Conference (EMTC '14), pp. 1-6, Naples, Italy, November 2014.

[10] N. Saquib, E. Hossain, and D. I. Kim, "Fractional frequency reuse for interference management in LTE-advanced hetnets," IEEE Wireless Communications, vol. 20, no. 2, pp. 113-122, 2013.

[11] T. Zhou, Y. Chen, C. Jiang, and K. J. R. Liu, "Pricing game for time mute in femto-macro coexistent networks," IEEE Transactions on Wireless Communications, vol. 14, no. 4, pp. 2118-2130, 2015.

[12] P. Y. Kong and G. Karagiannidis, "Backhaul-aware joint traffic offloading and time fraction allocation for 5G HetNets," IEEE Transactions on Vehicular Technology, 2016.

[13] J. Fan, D. Lee, G. Y. Li, and L. Li, "Multiuser scheduling and pairing with interference mitigation for LTE uplink cellular networks," IEEE Transactions on Vehicular Technology, vol. 64, no. 2, pp. 481-492, 2015.

[14] C. Kosta, B. Hunt, A. U. Quddus, and R. Tafazolli, "On interference avoidance through Inter-Cell Interference Coordination (ICIC) based on OFDMA mobile systems," IEEE Communications Surveys and Tutorials, vol. 15, no. 3, pp. 973-995, 2013.

[15] Y. Zhou, L. Liu, H. Du, L. Tian, X. Wang, and J. Shi, "An overview on intercell interference management in mobile cellular networks: from $2 \mathrm{G}$ to $5 \mathrm{G}$," in Proceedings of the IEEE International Conference on Communication Systems (ICCS '14), pp. 217-221, Macau, China, November 2014.

[16] O. Asenov, P. Koleva, and V. Poulkov, "Heuristic approach to dynamic Uplink Power Control in LTE," in Proceedings of the 36th International Conference on Telecommunications and Signal Processing (TSP '13), pp. 235-238, IEEE, Rome, Italy, July 2013.

[17] Y. Wang and S. Venkatraman, "Uplink power control in LTE heterogeneous networks," in Proceedings of the IEEE Globecom Workshops (GC Wkshps '12), pp. 592-597, Anaheim, Calif, USA, December 2012.

[18] A. Afifi, K. M. F. Elsayed, and A. Khattab, "Interference-aware radio resource management framework for the 3GPP LTE uplink with QoS constraints," in Proceedings of the 18th IEEE Symposium on Computers and Communications (ISCC '13), pp. 000693-000698, Split, Croatia, July 2013.

[19] H. Burchardt, Z. Bharucha, G. Auer, and H. Haas, "Uplink interference protection and scheduling for energy efficient OFDMA networks," EURASIP Journal on Wireless Communications and Networking, vol. 2012, article 180, 2012.

[20] S. A. Ahmad and D. Datla, "Distributed power allocations in heterogeneous networks with dual connectivity using backhaul state information," IEEE Transactions on Wireless Communications, vol. 14, no. 8, pp. 4574-4581, 2015.

[21] M. Morita, T. Nobukiyo, and K. Hamabe, "Uplink power control method for LTE femtocells based on resource usage aggregation," in Proceedings of the IEEE 23rd International Symposium on Personal, Indoor and Mobile Radio Communications (PIMRC '12), pp. 442-447, Sydney, Australia, September 2012.

[22] H.-S. Jo, C. Mun, J. Moon, and J.-G. Yook, "Interference mitigation using uplink power control for two-tier femtocell networks," IEEE Transactions on Wireless Communications, vol. 8, no. 10, pp. 4906-4910, 2009. 
[23] 3GPP, "Evolved universal terrestrial radio access (E-UTRA)," Physical Layer Procedures 3GPP TS 36.213, 2014.

[24] H. Claussen, "Efficient modelling of channel maps with correlated shadow fading in mobile radio systems," in Proceedings of the IEEE 16th International Symposium on Personal, Indoor and Mobile Radio Communications (PIMRC '05), vol. 1, pp. 512-516, September 2005. 

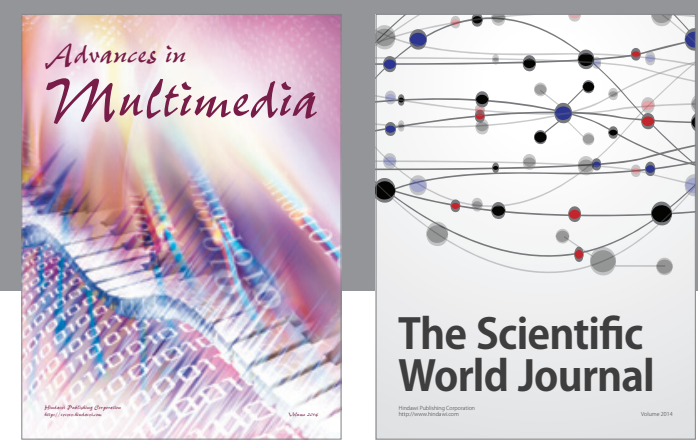

The Scientific World Journal
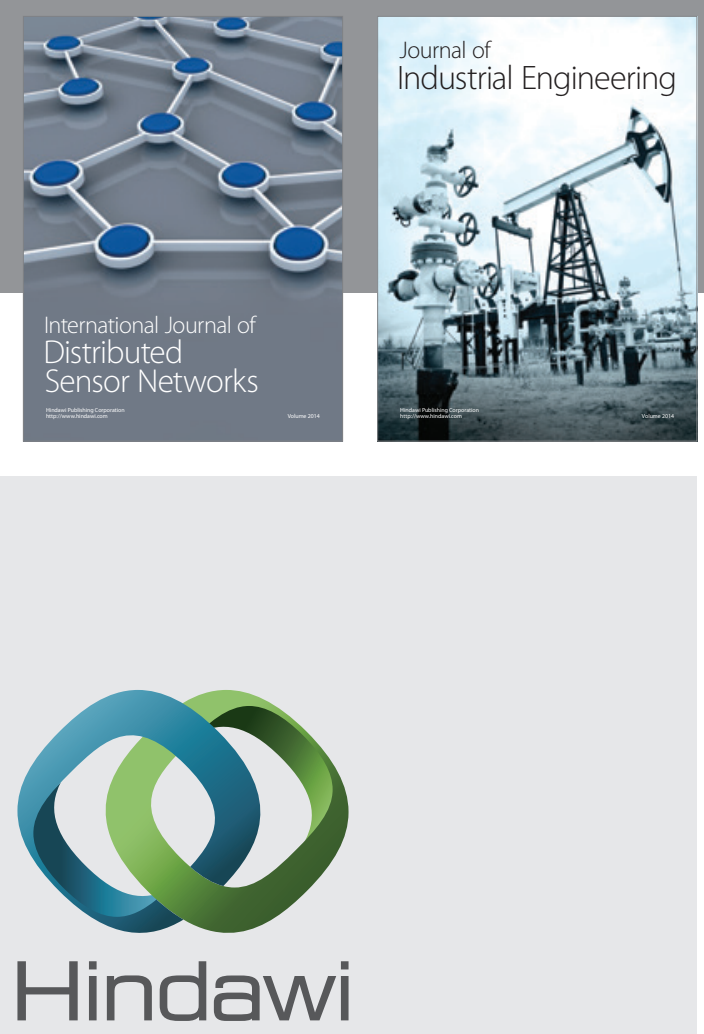

Submit your manuscripts at

http://www.hindawi.com

\section{Computer Networks} and Communications
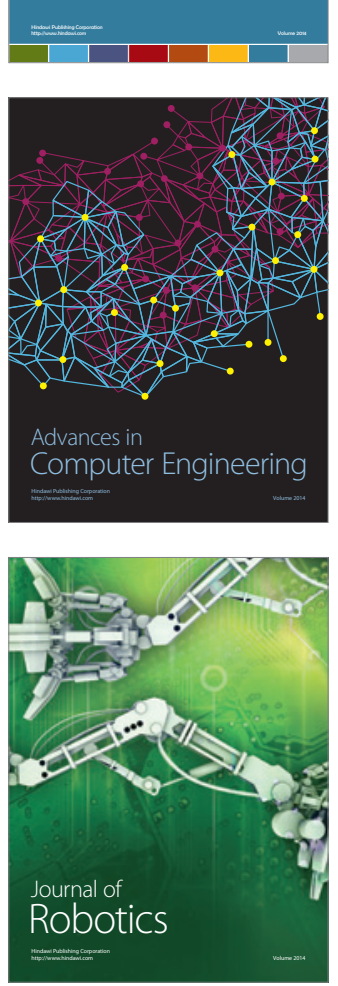
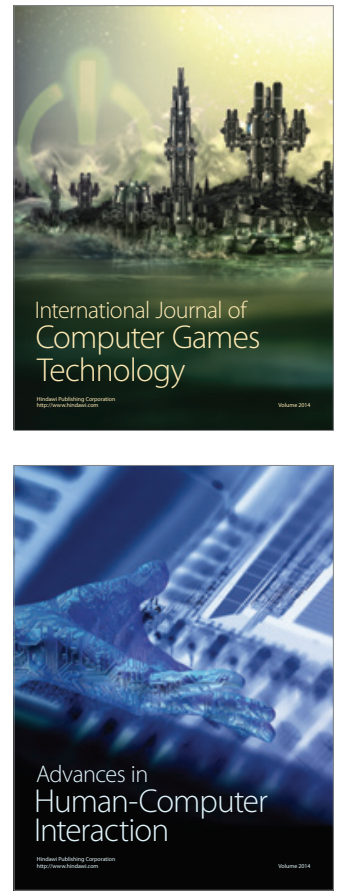
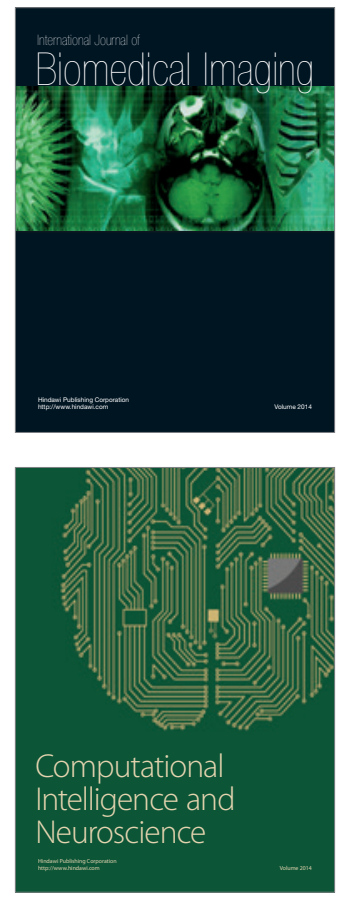
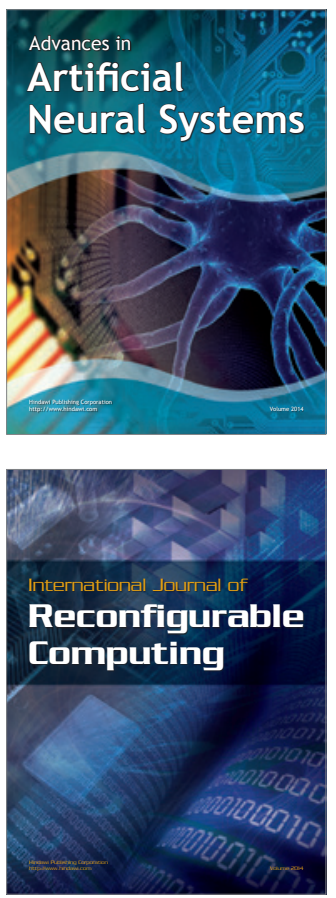
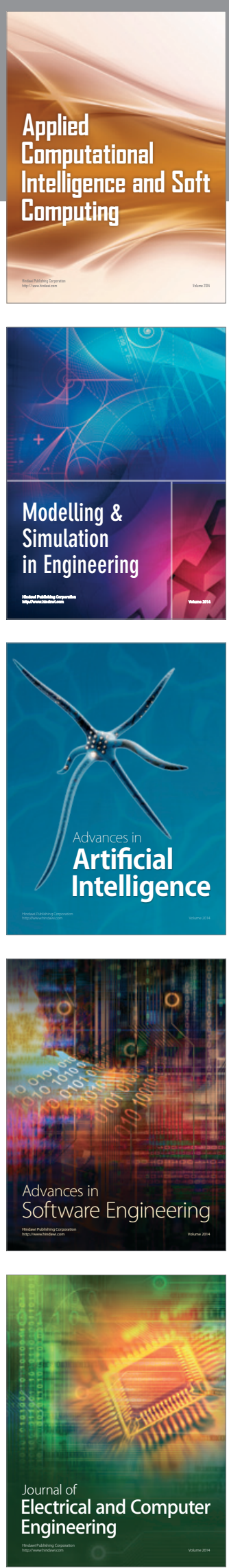